\title{
Emergence of Previously Unknown Poxviruses
}

Cynthia S. Goldsmith ${ }^{1}$, Maureen G. Metcalfe ${ }^{1}, \mathrm{Yu} \mathrm{Li}^{2}$ Whitni B. Davidson ${ }^{2}$, Kimberly Wilkins ${ }^{2}$ Amira A. Roess ${ }^{2}$, Lynda U. Osadebe ${ }^{2}$, Neil M. Vora ${ }^{2,3}$, Christopher H. Hsu ${ }^{2,3}$, Mary Reynolds ${ }^{2}$, Darin Carroll ${ }^{2}$, Inger K. Damon ${ }^{4}$, and Sherif R. Zaki ${ }^{1}$.

${ }^{1 .}$ Infectious Diseases Pathology Branch, ${ }^{2 .}$ Poxvirus and Rabies Branch, ${ }^{3 .}$ Epidemic Intelligence Services,

${ }^{4}$.Division of High-Consequence Pathogens and Pathology, Centers for Disease Control and Prevention, Atlanta, GA.

The virus family Poxviridae contains 4 genera whose members are able to infect humans: Orthopoxvirus, Parapoxvirus, Yatapoxvirus, and Molluscipoxvirus. The viruses causing smallpox and molluscum contagiosum are human pathogens, but the other poxviruses are zoonoses and humans are incidental hosts. Recently, several new species of zoonotic poxviruses have been recognized and characterized.

Two hunters, one in eastern Virginia and one in Connecticut, were cut on the fingers while field dressing deer [1]. Within 5-7 weeks, the cut areas had formed nodules and the patients sought medical attention. Biopsies of the skin lesions were taken and histopathological examination showed inclusions in the epidermis with pathological features consistent with a poxvirus infection. The biopsies were sent to the Centers for Disease Control and Prevention (CDC) for confirmation, and immunohistochemical (IHC) staining with anti-parapoxvirus antibodies was positive. Electron microscopic (EM) examination of the skin biopsies revealed ovoid poxvirus particles that were suggestive of parapoxviruses (Fig 1A). DNA was extracted from formalin-fixed, paraffin-embedded sections, and sequence analysis suggested a unique parapoxvirus strain that clusters with pseudocowpox viruses.

Another instance of poxvirus infections occurred that involved two patients [2]. An immuno-compromised patient from eastern Tennessee developed multiple lesions on her face, and had had exposure to animals while working at a horse stable. Another patient in western Missouri had a lesion on her hand, and had worked with donkeys in Tanzania. Biopsies were taken and showed lesions typical of cutaneous poxvirus infection, and the biopsies were sent to CDC. IHC staining using an anti-orf virus was positive, and EM evaluation showed ovoid particles consistent with a poxvirus (Fig 1B, C). Sequence analysis of the DNA extracts indicated that the viruses were novel poxviruses closely related to each other, with $88 \%$ similarity to viruses within the Parapoxvirus genus and $78 \%$ similarity to the Molluscipoxvirus genus.

Two cattle herders in the Republic of Georgia developed lesions and were suspected of infection with cowpox virus [3]. DNA was extracted from material obtained by swabbing the lesions and PCR was positive for nonvariola orthopoxviruses. Further evaluation by PCR was unable to identify the species but sequencing of the amplified region showed $96 \%$ identity with monkeypox virus and $98 \%$ identity with cowpox virus. A viral isolate was obtained and electron microscopy found virions that were characteristic of orthopoxviruses (Fig 1D, E).

A 45 year old female from Alaska developed a lesion on her shoulder which became ulcerated. A swab from the lesion was collected and a viral isolate was obtained. qPCR detected an orthopoxvirus and sequencing analysis confirmed a novel strain that lies basal to all Old World poxviruses but separate from New World poxviruses. EM examination of the isolate showed poxvirus-infected cells with A-type inclusions (Fig 1F, G).

In the past, vaccination against smallpox would have elicited cross-reactive antibodies that would likely have protected the patients in the orthopoxvirus cases. However, vaccination ceased in the 1970s, and as the population of naïve individuals increases, other previously unknown poxviruses may continue to emerge.

\section{References:}

[1] AA Roess et al, NEJM 363 (2010), p. 2621.

[2] LU Osadebe et al, CID 60 (2015), p. 195.

[3] NM Vora et al, NEJM 372 (2015), p. 1223. 

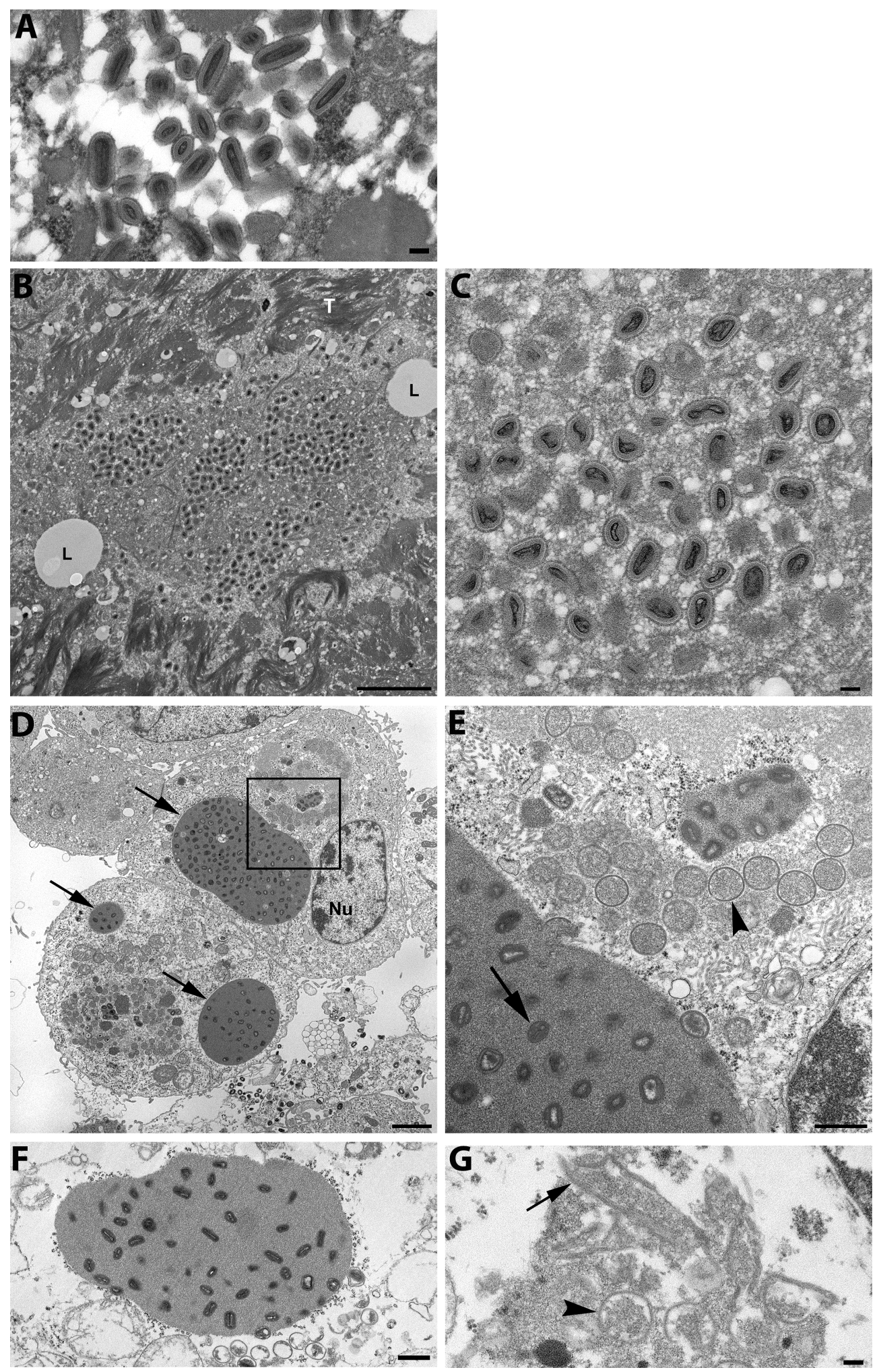

Figure 1. A) Biopsy of skin showing particles consistent with parapoxvirus. Bar, $100 \mathrm{~nm}$. B) Low magnification image of skin with a poxvirus-infected cell. L, lipid; T, tonofilaments. Bar, $2 \mu \mathrm{m}$. C) Higher magnification image of the same cell showing numerous virions. Bar, $100 \mathrm{~nm}$. D) Poxvirus-infected cell culture; arrows point to Atype inclusions. Higher magnification of the boxed area seen in (E). Bar, $2 \mu \mathrm{m}$. E) Mature virions (arrow) in a protein matrix of the A-type inclusion. Immature particles (arrowhead) are seen in a viral factory. Bar, $500 \mathrm{~nm}$. F) A-type inclusion from culture isolate, surrounded by ribosomes. Bar, $500 \mathrm{~nm}$. G) Viral factory, containing immature particles (arrowhead) and tubules (arrow) of unknown function. Bar, $100 \mathrm{~nm}$. 